\title{
Differential effects of explicit form-focused instruction on morphosyntactic development
}

\section{Hainu Xu \& Roy Lyster}

To cite this article: Hainu Xu \& Roy Lyster (2014) Differential effects of explicit form-focused instruction on morphosyntactic development, Language Awareness, 23:1-2, 107-122, DOI: 10.1080/09658416.2013.863899

To link to this article: http://dx.doi.org/10.1080/09658416.2013.863899

册 Published online: 05 Feb 2014.

Submit your article to this journal $\pi$

Џlll Article views: 384

Q View related articles ¿

View Crossmark data $₫$ 


\title{
Differential effects of explicit form-focused instruction on morphosyntactic development
}

\author{
Hainu $\mathrm{Xu}^{\mathrm{a} *}$ and Roy Lyster ${ }^{\mathrm{b}}$ \\ ${ }^{a}$ English Language Department, School of Foreign Languages, Nanjing University of Chinese \\ Medicine, 138 Xianlin Road, Nanjing 210023, China; ${ }^{b}$ Department of Integrated Studies in \\ Education, McGill University, 3700 McTavish Street, Montreal, QC H3A 1Y2, Canada
}

(Received 28 December 2012; accepted 27 September 2013)

\begin{abstract}
This study explores whether and to what degree explicit form-focused instruction (FFI) facilitates the use of morphosyntactic forms in second language oral production and also whether it has differential effects on morphosyntactic forms with different linguistic variables. Twenty-seven university-level Chinese EFL participants were randomly assigned to a control group and an experimental group. While the control group watched a television episode in English, the experimental group completed two form-focused activities (rule review and self-correction) designed to draw their attention to noun plural, past tense, and third-person singular in English in their oral production. All participants completed oral production pre- and post-test measures, the results of which showed that explicit FFI promoted the use of the target forms and that the facilitative effects were dependent on the complexity and regularity of the morphosyntactic forms. Regular (as opposed to irregular) and more complex morphosyntactic forms appeared to benefit more significantly from explicit FFI.
\end{abstract}

Keywords: form-focused instruction; morphosyntactic development; complexity; regularity

\section{Introduction}

Form-focused instruction refers to 'any pedagogical effort which is used to draw the learners' attention to form implicitly or explicitly' (Spada, 1997, p. 73). Explicit formfocused instruction (FFI), in which 'learners are encouraged to develop metalinguistic awareness of the rule' (Ellis, 2008, p. 438), has generally been found to be beneficial to second language acquisition (SLA) (Ellis, 2008; Norris \& Ortega, 2000; Spada, 2011; Spada \& Tomita, 2010). However, results of empirical studies suggest that explicit FFI may have differential effects on different language domains; that is, in comparison with lexical items, grammatical forms might be less susceptible to explicit FFI. Of those that reported facilitative effects of explicit FFI on the development of grammatical forms, some studies again have revealed differential effects on different grammatical forms (e.g. Mackey, 2006; Shook, 1994; Williams \& Evan, 1998; Yang \& Lyster, 2010). Researchers have proposed that linguistic variables such as complexity and regularity of the targeted grammatical forms are likely to moderate the efficacy of explicit FFI.

Morphosyntactic forms have long been recognised as the most difficult grammatical forms for second language (L2) learners mainly because of their low salience (Goldschneider \& DeKeyser, 2001; Mackey, 2006) and lack of communicative value (Han, 2004). Pedagogical efforts to help L2 learners acquire morphosyntactic forms have

*Corresponding author. Email: hainuxu@njutcm.edu.cn 
generated mixed results and researchers have pointed to the linguistic features of target forms as an important factor for explicit FFI. Few empirical studies, however, have directly investigated morphosyntactic forms with variable complexity and regularity.

\section{Previous research}

\section{The effect of explicit FFI in relation to complexity}

Research exploring the connection between the complexity of target linguistic structures and explicit FFI reflects two dominant points of view. One proposal is that explicit FFI should be more effective with more complex structures because they are more salient and thus more likely to be noticed and learned (DeKeyser, 2005; Hulstijn \& de Graaff, 1994; Schmidt, 1990, 2001). Another proposal is that more complex structures may be less likely to be learned than simpler ones because complex structures demand more attention (possibly creating a cognitive overload) in sentence processing and these attentional constraints may have a negative impact on L2 learning (e.g. McLaughlin \& Heredia, 1996). Results of empirical studies of attention and the learning of complex versus simple grammatical structures are also divided, arguably owing in part to the lack of consensus in how to conceptualise complexity and also from the inconsistency in its operationalisation (Housen, Pierrard, \& Van Daele, 2005; Spada \& Tomita, 2010).

In a quasi-experimental study investigating the effects of explicit instruction on pseudo-clefts of location (hard rule) and subject-verb inversion (easy rule), Robinson (1996) found an advantage for the explicitly instructed learners in their learning of the easy rule. Similarly, in DeKeyser's (1995) comparison of two pedagogical interventions (explicit-deductive vs. implicit-inductive) on categorical rules and prototypicality patterns in a miniature linguistic system, the explicit-deductive learning condition resulted in significant linguistic gains for categorical rules (easy), whereas the prototypicality patterns (hard) were more susceptible to the implicit-inductive condition. The learners in Williams and Evans' (1998) study, after a focus-on-form treatment, improved their performance on participial adjectives, but not on passive forms, which, according to the authors, are more complex. Results of these three studies indicate that explicit FFI may be more effective with less complex structures. Ellis (2002) made a similar suggestion in his review of 11 form-focused instruction studies conducted in the 1990s.

However, Uggen (2012) reported an opposite trend in a replication of Izumi and Bigelow's (2000) study. Investigating the function of output enhancement on the learning of complex and simple linguistic structures in English, Uggen (2012) found that learning was greater with the (more complex) past hypothetical conditional than with the (less complex) present hypothetical conditional. The author speculated that the complexity of the structure caused greater attention and more processing in working memory, which in turn benefitted L2 learning.

A third finding, however, is that explicit FFI has no differential effects on different grammatical forms. Housen et al. (2005) conducted a classroom study to investigate the effects of explicit instruction on passive forms and sentence negation in French, hypothesising that explicit instruction would work best for the more complex feature (passive). However, the statistical analysis indicated no significant difference between the two forms. de Graaff (1997) investigated the interaction between explicit instruction and the linguistic variables of complexity and the morphology/syntax distinction in the acquisition of four structures in eXperanto. The statistical results did not support his hypothesis that explicit instruction would be more effective with complex structures. de Graaff 
suggested that to better capture the differential effects of explicit instruction on linguistic structures, semantic salience should be taken into consideration in defining complexity.

In addition to studies with a clear definition of complexity, there have also been studies that found differential effects on different grammatical forms while drawing on constructs such as salience or meaningfulness to account for the differences. Shook (1994, 1999) investigated the effect of written input enhancement on L2 Spanish learners' development with respect to the present perfect and relative pronouns. The effect of his treatment was more significant in the 'more-meaningful' present perfect than in the 'lessmeaningful' relative pronoun (Shook, 1999, p. 45). In Mackey's (2006) study of the relationships between interactional feedback, learners' noticing of L2 forms, and their subsequent L2 development, the provision of feedback led to high levels of noticing and development for question forms but not for the other two grammatical forms under investigation (i.e. past tense and plural), owing arguably to the greater salience and communicative value of question forms in classroom discourse.

\section{The effect of explicit FFI in relation to regular and irregular past-tense forms}

Due to its morphological realisation, English past tense has remained a topic of theoretical debate centring on its representation and processing. There are two divergent views on the issue. One is the connectionist or single-system account (Rumelhart \& McClelland, 1986), according to which both regular and irregular forms of past tense are learned and computed in associative memory. The other is the symbolic or dual-system view (Pinker \& Prince, 1988; Pinker \& Ullman, 2002), according to which regular and irregular pasttense forms are stored and processed in two different systems. Irregular past-tense forms are stored in the lexicon, a division of declarative memory, whereas regular forms are computed by a rule relying on the procedural system. As Pinker and Ullman (2002) put it, 'irregular forms are just words, acquired and stored like other words, but with a grammatical feature like "past tense" incorporated into their lexical entries. Regular forms, by contrast, can be productively generated by a rule, just like phrases and sentences' (p. 456). This second dual-system view has been echoed by SLA researchers such as Skehan (1998) who proposed a dual-mode representational system consisting of two interrelated subsystems: an analytic rule-based system and a memory-driven exemplar-based system.

Along with the theoretical debates, some empirical studies have investigated the effects of intervention on the development of regular and irregular past-tense forms. Ellis (1987) explored how the amount of planning time available to L2 English learners in different tasks affected their use of three morphosyntactic forms (i.e. regular past tense, irregular past tense, and past copula), and found that attention to form (with more planning time) directly affected regular past-tense forms but not so much for the irregular past-tense forms.

Yang and Lyster (2010) investigated the effect of FFI (operationalised as production practice with different types of feedback) on the acquisition of regular and irregular pasttense forms. The effects of prompts (a more salient correction strategy) were larger than those of recasts for increasing accuracy in the use of regular past-tense forms, whereas prompts and recasts had similar effects on improving accuracy in the use of irregular past-tense forms. The authors suggested that the differing representational features of regular and irregular past-tense forms may have mediated the effects of feedback and also that the differential effects were in part due to the differing level of saliency between regular and irregular past-tense forms. 


\section{Research questions}

We designed the present study to address the following research questions:

(1) To what extent can explicit FFI affect the oral production of plural, past-tense, and third-person singular forms by university-level Chinese EFL learners?

(2) Are there differential effects of explicit FFI on the use of morphosyntactic forms varying in complexity?

(3) Are there differential effects of explicit FFI on the use of regular and irregular morphosyntactic forms?

\section{Method \\ Participants}

The participants were 27 Mandarin-speaking students (three males and 24 females) from a first-year English-major class at a university in Nanjing, China. They were 19-20 years old and had been learning English in classroom contexts for nine years. Based on the class list, we divided these students into two groups: experimental $(n=15)$ and control $(n=$ 12). Their English proficiency was measured by averaging their scores in the National College Entrance English Test and the Comprehensive English course grades (comprising vocabulary, grammar, reading, writing, listening, and speaking) prior to the study. An independent-samples $t$ test revealed no significant difference between the two groups in English proficiency, $t(25)=.008, p=.994$.

\section{Target forms and their complexity}

We chose three English morphosyntactic forms - noun plural, past tense, and third-person singular - as the target structures for several reasons: (1) the reported learning difficulty for Chinese learners; (2) their absence in the participants' L1 Chinese, thus controlling for L1 transfer effects; (3) their inclusion of three morphosyntactic features (tense, person, and number); and (4) the inclusion of both regular and irregular forms in the case of past tense. In addition, these three forms represent differing levels of complexity as illustrated next.

In previous studies, researchers have defined complexity mainly from three different perspectives: psycholinguistic, linguistic, and pedagogical, each with its merits and flaws (see Spada \& Tomita, 2010). In the present study, we operationalise complexity in a hybrid manner, taking into account psycholinguistic, cognitive, and linguistic factors, as has been done in the L2 English morpheme acquisition studies. According to Goldschneider and DeKeyser's (2001) meta-analysis of the morpheme acquisition studies, the three target forms of the present study follow a relative rank order of acquisition: plural is acquired earlier than past tense (regular -ed form) which is then followed by third-person singular. Of the five determinants (i.e. perceptual salience, semantic complexity, morphophonological regularity, syntactic category, and frequency) proposed by Goldschneider and DeKeyser, these target forms have equal values on almost all the determinants except semantic complexity. They follow a rank order of semantic complexity consistent with the acquisition order (see Table 1), suggesting that semantic complexity could be a chief contributing factor for the observed order. Other contributing factors proposed by researchers include processing constraints (Pienemann, 1989, 1998), formalsemantic redundancy (Han, 2004; VanPatten, 1996), morpheme agreement distance 


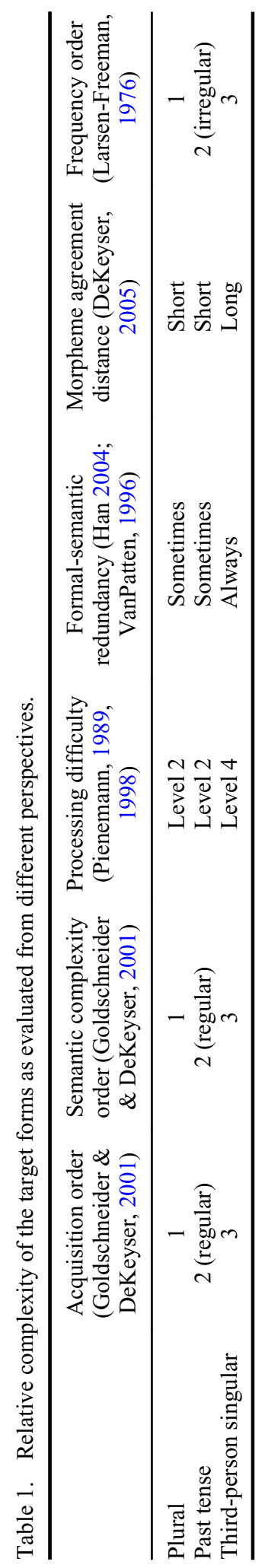


(DeKeyser, 2005), and frequency (Larsen-Freeman, 1976). Based on the relative acquisition order and the relative values (or order) of the target forms in terms of those contributing factors, we operationalised the complexity of the target forms as follows: third-person singular is more complex than past tense which is more complex than noun plural.

\section{Explicit FFI treatment}

According to Ellis (2008), one type of explicit FFI that is especially facilitative is metalinguistic activity such as providing metalinguistic information to the learners and encouraging reflection on and self-repair of their errors. Metalinguistic activity entails awareness both at the level of noticing and at the level of understanding (Schmidt, 2001), so it may foster the development of both explicit and implicit L2 knowledge. It has also been suggested that metalinguistic activities can be effective only when they are combined with other communicative practice activities. Guided by these principles, we employed two metalinguistic activities (rule review and self-correction) in conjunction with an oral production activity.

Oral production is claimed to draw learners' attention to the L2 structures they produce and also to help learners notice what they are unable to produce (Swain, 1995). However, production may not always succeed in drawing learners' attention to target forms (Izumi \& Bigelow, 2000) and therefore extended opportunities are necessary. In the present study, the learners had an opportunity, immediately following the oral production, to review the basic rules concerning the target forms. This rule review activity was designed to activate learners' metalinguistic knowledge and to reinforce their rule understanding with a view to strengthening existing knowledge representations.

Drawing on Swain's (1995) output hypothesis, Ellis (2003) identified one more role of output in L2 acquisition: output provides the learner with 'auto-input' in the sense that learners can attend to the input provided by their own production (see also Lynch, 2001). In view of the potential effects of auto-input, this study made use of the learners' output as input for further activities. The participants were directed to listen to their own previously recorded oral production and correct their errors concerning the target forms. This self-correction task was designed to orient learners' attention to problematic features by providing opportunities for cognitive reprocessing or cognitive comparison (Ellis, 2002). During the activity, learners reflected on their output and considered ways of modifying it to improve accuracy in relation to the targeted morphosyntactic forms.

\section{Procedure}

The study was conducted in two language laboratories where the first author worked with the experimental group and the regular course instructor with the control group. For the experimental group, there were five main stages of activities: (1) Production Task 1 (as pre-test), (2) Rule Review, (3) Self-correction, (4) Production Task 2 (as immediate posttest), and (5) Production Task 3 (as delayed post-test). The control group followed the same procedure except at stages (2) and (3). While the experimental group was reviewing grammar rules and correcting errors, the control group watched an episode from the American TV series Friends. The details of the five stages are illustrated as follows.

\section{Stage 1: Production Task 1}

The researcher and the course instructor each gave their respective group a task card telling them to prepare (in five minutes) an oral description (4-5 minutes long) of a friend. 
The content requirement included the person's appearance, personality, and daily routine, which created obligatory contexts for using noun plurals and third-person singular forms. The task also required the students to tell a funny story about the friend, which was to elicit the use of past-tense forms. Then they completed the oral production task, which they recorded and saved on the computer using the Lange Digital Language Laboratory program.

\section{Stage 2: Rule review}

After Production Task 1, there was a 10-minute rule review session for the experimental group where the researcher reviewed the basic rules concerning the use of noun plural, past tense, and third-person singular. She showed some illustrative sentences containing typical errors made by Chinese students in regard to the three target forms on a projector and also explained in Chinese as to why the sentences were wrong. Regarding the past tense, rules of use were reviewed in general, but the illustrative sentences contained errors of both regular and irregular past-tense forms.

\section{Stage 3: Self-correction}

After the rule review, the students listened to their previously recorded oral production at their own pace in order to identify and correct their errors concerning the use of those target forms. The researcher gave them a worksheet for taking notes of the errors identified, the correct forms, and also the reasons for their correction. These notes were collected for later analysis. The self-correction task lasted about 20 minutes.

\section{Stage 4: Production Task 2}

Following the self-correction task, there was a second oral production task that was similar to Production Task 1 in requirement and in delivery format, but not in terms of topic. Participants were asked to describe a family member, instead of a friend, and also to tell a touching story about the person. Production Task 2 was also recorded for later analysis.

\section{Stage 5: Production Task 3}

Twenty-six days after the treatment, the participants finished a third oral production task. It had the same requirement and delivery format as the first two oral production tasks except that the topic of the talk was changed to be about a teacher. The participants were required to tell a funny, touching, or embarrassing story about the teacher. Production Task 3 was also recorded for analysis.

Besides the five major stages of activities, there were also two qualitative data collection activities following the second oral production task, namely a questionnaire survey and retrospective interviews. Two different questionnaires were used for the experimental and control groups. For the experimental group, five closed-item questions asked the participants to indicate their degree of focal attention on the three target forms during the first two oral tasks, with some open space for writing reasons for focusing attention on a certain target form in a task. For the control group, care was taken not to mention the target forms. These participants were asked to indicate which of the five aspects (content, vocabulary, fluency, pronunciation, or grammar) their attention was focused on in the two oral tasks. After the questionnaire session, the researcher interviewed three 
participants from the experimental group for collecting more in-depth information about their attentional focus during the oral tasks. She also interviewed two participants from the control group with general questions such as their perception of the purpose of the activities and of their performance in relation to grammar.

\section{Data analysis}

The first author transcribed the participants' oral protocols from the three production tasks. After verification of all the transcripts, she coded them for the obligatory contexts of each target form and accurate occurrences of the forms in those obligatory contexts. A second coder coded $10 \%$ of the protocols. Measured with Cohen's kappa test, the intercoder reliability was found to be $k=.897$ ( $p<.001), 95 \%$ CI [0.504, 0.848], indicating a high level of agreement.

The accuracy rate of each participant for using each target form was calculated by dividing the total number of accurate occurrences by the total number of obligatory contexts. This occurrence-in-obligatory-contexts scoring method was used following the practice in most of the acquisition order studies (see the review by Goldschneider \& DeKeyser, 2001) and in some previous FFI studies of morphological acquisition (e.g. Pawlak, 2008; Yang \& Lyster, 2010).

The accuracy scores were first submitted to a between-group analysis of variance with a Mann-Whitney U test. Then a Wilcoxon test was run for a within-group analysis for the experimental group and the control group separately to determine to what extent each group varied in their accuracy in the three oral production tasks. A series of Wilcoxon tests were also performed for a within-group analysis of the experimental group to determine whether the participants had benefitted from the explicit FFI activities to the same extent on all three forms. Cohen's $d$ was calculated to measure the magnitude of effects of the treatment with respect to between-group and within-group contrasts. Following Oswald and Plonsky's (2010) suggestions for SLA standards, effect sizes were classified as small $(d<0.7)$, medium $(0.7 \leq d<1)$, or large $(d \geq 1)$.

The self-correction notes were analysed by tallying the number of errors identified and accurately corrected for each target form. The questionnaires and interviews were also analysed as supplementary evidence for the participants' focus of attention in different tasks.

\section{Results}

\section{Overall effects}

The descriptive statistics concerning the accuracy scores of the experimental and control groups appear in Table 2. As for the between-group analysis, the Mann-Whitney U test confirmed that there was no significant difference at the time of pre-testing but revealed a significant difference with a medium effect between the two groups at the time of immediate post-testing, $U=46.0, z(25)=-2.149, p=.032, d=0.93$. There was no significant difference between the two groups in their accuracy at the time of delayed post-testing. As for the within-group analysis comparing pre-test and immediate post-test scores, the Wilcoxon tests confirmed that the experimental group's increase in accuracy was significant with a large effect size, $z=-2.919, p=.004, d=1.00$, and revealed no significant difference in the control group's performance over time, $z=-1.334, p=.182$. 


\section{Effects on morphosyntactic forms varying in complexity}

Table 3 displays the accuracy scores of the experimental group in using the three different morphosyntactic forms during the three oral tasks. There was an increase for all the three forms at the immediate post-test. Although the improvement tendency was not maintained at the delayed post-test, the scores of all three forms were still higher at the delayed post-test than at the pre-test. As shown in Table 4, the Wilcoxon tests revealed a significant accuracy increase between the pre- and post-test for third-person singular with a large effect size, $z=-2.449, p=.014, d=1.05$, but not for the other two forms.

\section{Effects on regular and irregular morphosyntactic forms}

Table 5 presents the experimental group's accuracy scores in using regular and irregular past-tense forms in the three oral tasks. The descriptive statistics show an increase in

Table 2. Descriptive statistics of both groups' overall accuracy scores.

\begin{tabular}{lcccccc}
\hline & & $n$ & Mean & Minimum & Maximum & SD \\
\hline Experimental & Pre-test & 15 & .748 & .389 & 1.000 & .139 \\
& Post-test & 15 & .873 & .658 & 1.000 & .109 \\
\multirow{3}{*}{ Control } & Delayed & 14 & .844 & .717 & .944 & .070 \\
& Pre-test & 12 & .795 & .577 & .944 & .114 \\
& Post-test & 12 & .765 & .567 & .960 & .123 \\
& Delayed & 11 & .825 & .556 & .964 & .154 \\
\hline
\end{tabular}

Table 3. Descriptive statistics of the experimental group's accuracy scores for each form.

\begin{tabular}{llccccc}
\hline & & $n$ & Mean & Min. & Max. & SD \\
\hline Plural & Pre-test & 15 & .831 & .000 & 1.000 & .316 \\
& Post-test & 15 & .940 & .667 & 1.000 & .095 \\
Past tense & Delayed & 14 & .930 & .786 & 1.000 & .067 \\
& Pre-test & 15 & .719 & .000 & 1.000 & .304 \\
Third-person singular & Post-test & 15 & .888 & .600 & 1.000 & .120 \\
& Delayed & 13 & .774 & .500 & 1.000 & .156 \\
& Pretest & 15 & .643 & .429 & 1.000 & .185 \\
& Post-test & 15 & .830 & .455 & 1.000 & .171 \\
& Delayed & 14 & .681 & .000 & 1.000 & .309 \\
\hline
\end{tabular}

Table 4. Between-task contrasts and magnitude of effect sizes for each target form.

\begin{tabular}{lcccc}
\hline & & Post-test vs. Pre-test & Delayed vs. Post-test & Delayed vs. Pre-test \\
\hline Plural & $Z$ & -.980 & -.712 & -.204 \\
& $p$ & .327 & .476 & .838 \\
\multirow{4}{*}{ Past tense } & $d$ & .47 & -.12 & .43 \\
& $Z$ & -1.790 & -1.538 & .280 \\
\multirow{4}{*}{ Third-person singular } & $p$ & .074 & .12 & .78 \\
& $d$ & .73 & -.819 & .228 \\
& $Z$ & -2.449 & -1.647 & .534 \\
& $p$ & .014 & .099 & .15 \\
\hline
\end{tabular}


Table 5. Descriptive statistics of the experimental group's accuracy in using regular and irregular past-tense forms.

\begin{tabular}{llccccc}
\hline & & $n$ & Mean & Minimum & Maximum & SD \\
\hline \multirow{2}{*}{ Regular } & Pre-test & 15 & .628 & .000 & 1.000 & .435 \\
& Post-test & 15 & .874 & .33 & 1.000 & .215 \\
& Delayed & 12 & .738 & .000 & 1.000 & .290 \\
\multirow{4}{*}{ rregular } & Pre-test & 15 & .749 & .000 & 1.000 & .320 \\
& Post-test & 15 & .865 & .27 & 1.000 & .210 \\
& Delayed & 13 & .772 & .43 & 1.000 & .177 \\
\hline
\end{tabular}

Table 6. Between-task contrasts and magnitude of effect sizes for regular and irregular past-tense forms.

\begin{tabular}{lcccc}
\hline & & Post-test vs. Pre-test & Delayed vs. Post-test & Delayed vs. Pre-test \\
\hline Regular & $Z$ & -1.963 & -1.400 & -1.067 \\
& $p$ & .050 & .161 & .286 \\
& $d$ & .72 & -.53 & .30 \\
Irregular & $Z$ & -1.070 & -.944 & -.175 \\
& $p$ & .285 & .345 & .861 \\
& $d$ & .43 & -.48 & .09 \\
\hline
\end{tabular}

mean scores for both regular verbs (from $62.8 \%$ to $87.4 \%$ ) and irregular verbs (from $74.9 \%$ to $86.5 \%$ ) between the pre-test and immediate post-test. However, as shown in Table 6, it was only in the use of regular past-tense forms that a significant difference was detected between pre- and post-test accuracy scores and it was a marginal one with a medium effect size, $z=-1.963, p=.050, d=.72$.

\section{Discussion}

\section{Explicit FFI and morphosyntactic development}

The statistical analysis indicated that the experimental group performed significantly better than the control group at the immediate post-test, with a significant accuracy improvement in using the target forms, as one might expect, given the brief instructional treatment to which they were exposed for the purpose of supporting such improvement. Learners without such support but with additional exposure to input instead made no significant improvement. Although similar results are likely to occur in other EFL settings, we hasten to caution that, because of our use of non-parametric tests, our results demonstrate the effectiveness of explicit FFI for the sample in this particular EFL setting and cannot be generalised to other settings.

According to Ellis (2006), acquisition can be defined in three senses, one of which is increased control over forms that have been partially acquired. Under this definition, the results of the current study could be regarded as evidence for the facilitative effect of explicit FFI on the acquisition of L2 morphosyntactic forms for these EFL learners, because they demonstrated increased control over the targeted morphosyntactic forms. To measure the effect of explicit FFI, the current study employed free constructed response measures in the form of oral production tasks. When learners produce target 
forms in tasks involving spontaneous oral production, their unplanned language use is hypothesised to tap their implicit knowledge (Ellis, 2008). In this sense, the results of this study lend support to the proposal that explicit FFI can contribute both to learners' conscious knowledge of the target forms and to their ability in using those forms in unanalysed spontaneous ways (Spada, 2011).

Results of the current study can be compared with two previous explicit FFI studies. Muranoi (2000) found a positive and durable effect for interaction enhancement on the learning of English articles for Japanese learners. Pawlak (2008) reported significantly positive and lasting effects of corrective feedback on Polish learners' use of English third-person singular forms and attributed the favourable results to the instructional treatment, which combined output enhancement and recasting. The positive findings of the present study and those of Muranoi (2000) and Pawlak (2008) together underscore the importance of using a combination of instructional techniques in order for explicit FFI to achieve the desired effect on morphosyntactic forms.

However, the current study also differed from those of Muranoi (2000) and Pawlak (2008) in that it did not produce a lasting effect. This difference is perhaps due to the fact that the instructional treatment in the present study comprised only one 30-minute session, whereas Muranoi (2000) employed three 30-minute training sessions and Pawlak (2008) had four 20-minute treatment sessions. Therefore, caution is needed in interpreting the findings of the present study, given the short duration of the treatment and also the limited sample size. Noteworthy, however, is that the accuracy increase in this study was obtained at an exceptionally low price as measured by valuable classroom time. Another important point is the originality of using learners' output as the auto-input for further processing during explicit FFI activities. This kind of short and innovative pedagogical intervention can be especially useful and feasible in an EFL learning context with limited in-class exposure and large class sizes.

\section{Complexity and explicit FFI}

Whereas the descriptive statistics revealed an accuracy increase for all three morphosyntactic forms in the immediate post-test, the only statistically significant increase was for third-person singular, which yielded a large effect size. The increase for past-tense forms yielded a medium effect size, but the accuracy increase was not significant, thus necessitating a larger sample size to confirm the lack of randomness of the effect. Based on these observations, we speculate that the effect of explicit FFI may vary depending on the complexity of morphosyntactic forms, with more complex forms (such as third-person singular and, to a lesser extent, past-tense forms) being more susceptible than less complex forms (such as noun plurals) to explicit FFI activities, as suggested by DeKeyser (2005), Hulstijn and de Graaff (1994), and Schmidt (1990, 2001). This also corroborates findings from Uggen (2012) that form-focused activities help learners acquire complex morphosyntactic forms better.

One plausible explanation is the oft-mentioned ceiling effect for the less complex forms (noun plural). The accuracy rate for noun plural was as high as $84.1 \%$ at the pretest and so there was less potential for improvement, in contrast to the more complex form (third-person singular), which yielded lower accuracy scores at the pre-test and thus had more room for improvement. If this interpretation is correct, the implication is that more complex morphosyntactic forms are a better choice for explicit FFI activities; or, to be accurate, explicit FFI should target morphosyntactic forms that are best matched to the learners' knowledge base, neither too easy nor too difficult. 
The other explanation lies in the mediating effect of complexity on attentional processes during explicit FFI. It is likely that the more complex structures triggered more focused attention and more processing in working memory, which in turn contributed to L2 learning. This interpretation is based on the analysis of the qualitative data. The analysis of Production Task 1 and the self-correction notes revealed that, although the number of obligatory contexts was similar for all three target forms (120 for third-person singular, 114 for past tense and 118 for plural), more errors occurred for third-person singular and past tense than for noun plural (49 vs. 27 vs. 14). Accordingly, participants corrected a relatively larger number of errors for third-person singular and past tense than for noun plural ( 23 vs. 15 vs. 12 ) in the self-correction task. According to the questionnaire survey, eight out of the 15 participants claimed to have focused their attention on third-person singular and six of them focused their attention on past tense during the second oral production. The reason they gave for focusing more on the more complex target forms is that they were surprised by the large number of errors that they made during the first oral task and noticed during the self-correction session.

It would seem therefore that the self-correction activity was the stage at which the participants developed different levels of enhanced awareness towards the three target forms. A plausible explanation would be as follows. The more complex a certain morphosyntactic form is, the larger number of errors a learner may make on the form. The more errors the learner identifies and corrects for a target form, the more conscious attention the participant may pay to that item. Accordingly, the self-correction activity triggered more focal attention towards the more complex forms (especially third-person singular), which may be a major cause of the differential effects observed in this study.

Also noteworthy are the varying degrees in the decline of accuracy scores of the three forms from the immediate post-test to the delayed post-test. Third-person singular and past tense had a sharper accuracy decrease. This might be interpreted as another aspect of the effects of complexity. In the long run, the effects of explicit FFI may be better maintained with the less complex morphosyntactic forms (like noun plural). In other words, the effects on the more complex forms (like third-person singular), owing to their complexity as reflected in the higher degree of processing difficulty (Pienemann, 1989, 1998) and lower input frequency (Larsen-Freeman, 1976), may wear off more quickly as time goes by. If this is the case, to obtain a sustainable effect on more complex morphosyntactic forms, some immediate follow-up activities must be included in explicit FFI.

\section{Regularity and explicit FFI}

Although both regular and irregular past-tense forms improved under the explicit FFI treatment, only the regular forms showed a marginally significant effect $(p=.05)$ with a medium effect size, suggesting that regular and irregular morphosyntactic forms may benefit differently from explicit FFI, with regular forms benefitting to a greater extent. The present finding is in line with that of Ellis (1987) who also reported that attention to form affected regular past-tense forms more than irregular past-tense forms.

This result might be explained by the dual-mode representational system (Skehan, 1998). It is likely that the differing representational features of regular and irregular pasttense forms mediated the effects of explicit FFI, as was suggested in Yang and Lyster's (2010) study. Skehan submitted that interlanguage change is more effectively activated through the rule-based system and that conscious awareness predisposes learners towards such a rule-based perspective. In this vein, the processing of rule-based forms (regular 
past tense) might be more amenable to explicit FFI than the processing of exemplar-based forms (irregular past tense).

\section{Complexity and (or) regularity and explicit FFI}

Results of the current study suggest that complexity and regularity may both moderate the effects of explicit FFI on morphosyntactic forms, thus begging the question as to whether there is an interaction between complexity and regularity. While the current study cannot directly address this issue due to design limitations, it has generated some interesting results worth discussing.

Learners in this study improved significantly in their use of third-person singular but not as much in their use of past-tense forms, owing arguably to the greater complexity of third-person singular relative to past tense, which makes the former more amenable to explicit FFI. However, when the analysis of accuracy development in the use of pasttense forms distinguished between regular and irregular forms, significant improvement was found over time for regular but not irregular forms. This suggests that regularity may be a significant moderating factor for explicit FFI and that regular morphosyntactic forms may be more responsive to form-focused activities. It may be the case that third-person singular forms showed stronger effects because they are nearly all regular forms, whereas past tense did not show as much effect due to its greater proportion of irregular forms. In other words, the complexity effect of explicit FFI on third-person singular and past tense may turn out to be an effect of regularity.

Whatever the causes of the differential effects, the finding that the participants improved significantly on third person singular forms but not on past tense may still lend some empirical support to Hulstijn and de Graaff's (1994) hypothesis that 'The advantage of explicit instruction is greater when language production can only be based on rule application, than when it can be based not only on rule application but also on the retrieval of individually stored items (“item learning")' (p. 105).

\section{Conclusion}

This study has many limitations. First, the limited number of participants may have had an impact on the results. For example, the slight drop in the control group's accuracy score at the immediate post-test may have resulted from the small sample size, which allowed extreme cases to pull down the group mean to a significant extent. Although nonparametric statistical procedures were employed to minimise the effects of such a limitation, the generalisability of the study still remains a problem. Another limitation is related to the length of treatment and the short duration of the study. We suggest that future studies involve a larger sample size and extend the duration of the experiment so as to test the long-term effects of explicit FFI.

The above limitations notwithstanding, findings of the present study contribute to the existing body of literature on the positive role of explicit FFI in L2 morphosyntactic development. The study brings to the fore the complexity and regularity of the target forms as key variables influencing the effects of explicit FFI on morphosyntactic acquisition. This study also suggests some important areas worthy of further investigation. The study targeted three morphosyntactic forms and the learners decided which to focus on depending on their own individual and temporal learning needs. It would be appealing for future studies to disentangle the effects of form complexity from personal choice by having, for example, more experimental groups with just one morphosyntactic form per 
group. Other questions raised by this study worthy of further pursuit are whether there really exists an interaction between complexity and regularity of the target forms in FFI studies and whether learner variables such as L2 proficiency influence such an interaction.

\section{Acknowledgements}

This study was funded by a grant from the Jiangsu Social Sciences Fund (No. 11YYD019) and the State Scholarship Fund of China (No. 2011832579). The paper was presented at the 11th International Conference of the Association for Language Awareness at Concordia University on 9 July 2012. We thank the co-editors and the reviewers for their helpful comments and suggestions.

\section{Notes on contributors}

Dr Hainu Xu is currently an associate professor at the English Language Department, School of Foreign Languages, Nanjing University of Chinese Medicine. Her research interests include formfocused instruction, L2 English morphosyntactic development, and ELT material development and evaluation.

Dr Roy Lyster is currently a professor at the Department of Integrated Studies in Education, McGill University. His research examines content-based second language instruction and the effects of instructional interventions - such as teacher scaffolding and corrective feedback - designed to counterbalance form-focused and content-based approaches. His research interests also include collaboration among language teachers for integrated language learning and biliteracy development.

\section{References}

de Graaff, R. (1997). The eXperanto experiment: Effects of explicit instruction on second language acquisition. Studies in Second Language Acquisition, 19, 249-297.

DeKeyser, R. (1995). Learning second language grammar rules: An experiment with miniature linguistic system. Studies in Second Language Acquisition, 17, 379-410. doi: http://dx.doi.org/ $10.1017 / \mathrm{S} 027226310001425 \mathrm{X}$

DeKeyser, R. (2005). What makes learning second language grammar difficult? A review of issues. Language Learning, 55(Supplement 1), 2-25. doi: 10.1111/j.0023-8333.2005.00294.x

Ellis, R. (1987). Interlanguage variability in narrative discourse: Style shifting in the use of the past tense. Studies in Second Language Acquisition, 9, 1-20. doi: http://dx.doi.org/10.1017/ S0272263100006483

Ellis, R. (2002). The place of grammar instruction in the second/foreign curriculum. In E. Hinkel \& $\mathrm{S}$. Fotos (Eds.), New perspectives on grammar teaching in second language classrooms (pp. 17-34). Mahwah, NJ: Lawrence Erlbaum.

Ellis, R. (2003). Task-based learning and teaching. Oxford: Oxford University Press.

Ellis, R. (2006). Researching the effects of form-focused instruction on L2 acquisition. AILA Review, 19, 18-41. doi: 10.1075/aila.19.04ell

Ellis, R. (2008). Explicit form-focused instruction and second language acquisition. In B. Spolsky \& F.M. Hult (Eds.), The handbook of educational linguistics (pp. 437-455). Oxford: Blackwell.

Goldschneider, J.M., \& DeKeyser, R.M. (2001). Explaining the "natural order of L2 morpheme acquisition' in English: A meta-analysis of multiple determinants. Language Learning, 51, 1-50. doi: 10.1111/1467-9922.00147

Han, Z.H. (2004). Fossilization in adult second language acquisition. Clevedon: Multilingual Matters.

Housen, A., Pierrard, M., \& Van Daele, S. (2005). Rule complexity and the efficacy of explicit grammar instruction. In A. Housen \& M. Pierrard (Eds.), Investigations in instructed second language acquisition (pp. 235-269). Amsterdam: Mouton de Gruyter.

Hulstijn, J.H., \& de Graaff, R. (1994). Under what conditions does explicit knowledge of a second language facilitate the acquisition of implicit knowledge? A research proposal. AILA Review, 11, 97-112. Retrieved from http://www.aila.info/en/publications/aila-review/volumes-1-15/73aila-review-issue-11.html 
Izumi, S., \& Bigelow, M. (2000). Does output promote noticing and second language acquisition? TESOL Quarterly, 34, 239-278. Retrieved from http://www.jstor.org/stable/3587952

Larsen-Freeman, D. (1976). An explanation for the morpheme acquisition order of second language learners. Language Learning, 26, 125-134. doi: 10.1111/j.1467-1770.1976.tb00264.x

Lynch, T. (2001). Seeing what they meant: Transcribing as a route to noticing. ELT Journal, 55(2), 124-132. doi: 10.1093/elt/55.2.124

Mackey, A. (2006). Feedback, noticing and instructed second language learning. Applied Linguistics, 27, 1-27. doi: 10.1093/applin/ami051

McLaughlin, B., \& Heredia, R. (1996). Information-processing approaches to research on second language acquisition and use. In W. Ritchie \& T. Bhatia (Eds.), Handbook of second language acquisition (pp. 213-228). San Diego, CA: Academic Press.

Muranoi, H. (2000). Focus on form through interaction enhancement: integrating formal instruction into a communicative task in EFL classrooms. Language Learning, 50(4), 617-673. doi: $10.1111 / 0023-8333.00142$

Norris, J., \& Ortega, L. (2000). Effectiveness of L2 instruction: A research synthesis and quantitative meta-analysis. Language Learning, 50, 417-528. doi: 10.1111/0023-8333.00136

Oswald, F.L., \& Plonsky, L. (2010). Meta-analysis in second language research: Choices and challenges. Annual Review of Applied Linguistics, 30, 85-110. doi: http://dx.doi.org/10.1017/ S0267190510000115

Pawlak, M. (2008). The effect of corrective feedback on the acquisition of the English third-person -s endings. In D. Gabrys-Barker (Ed.), Morphosyntactic issues in second language acquisition (pp. 187-202). Clevedon: Multilingual Matters.

Pienemann, M. (1989). Is language teachable? Psycholinguistic experiments and hypotheses. Applied Linguistics, 10(1), 52-79. Retrieved from http://eric.ed.gov/?id=EJ385570

Pienemann, M. (1998). Language processing and second language development: Processability theory. Amsterdam: Benjamins.

Pinker, S., \& Prince, A. (1988). On language and connectionism: Analysis of a parallel distributed processing model of language acquisition. Cognition, 28, 73-193.

Pinker, S., \& Ullman, M. (2002). The past and future of the past tense. Trends in Cognitive Sciences, 6, 456-463. doi: http://dx.doi.org/10.1016/S1364-6613(02)01990-3

Robinson, P. (1996). Learning simple and complex second language rules under implicit, incidental, rule-search, and instructed conditions. Studies in Second Language Acquisition, 18, 27-67. doi: http://dx.doi.org/10.1017/S0272263100014674

Rumelhart, D.E., \& McClelland, J.L. (1986). On learning the past tenses of English verbs. In J.L. McClelland, D.E. Rumelhart, \& the P.D.P. Research Group (Eds.), Parallel distributed processing: Explorations in the microstructures of cognition: Vol. 2. Psychological and biological models (pp. 216-271). Cambridge, MA: MIT Press.

Schmidt, R. (1990). The role of consciousness in second language learning. Applied Linguistics, 11, 129-158. doi: 10.1093/applin/11.2.129

Schmidt, R. (2001). Attention. In P. Robinson (Ed.), Cognition and second language instruction (pp. 3-32). Cambridge: Cambridge University Press.

Shook, D. (1994). FL/L2 reading, grammatical information, and the input-to-intake phenomenon. Applied Language Learning, 52, 57-93. Retrieved from http://eric.ed.gov/?id=EJ508428

Shook, D. (1999). What foreign language reading recalls reveal about the input-to-intake phenomenon. Applied Language Learning, 10(1\&2), 39-76. Retrieved from http://eric.ed.gov/? id=EJ605099

Skehan, P. (1998). A cognitive approach to language learning. Oxford: Oxford University Press.

Spada, N. (1997). Form-focused instruction and second language acquisition: A review of classroom and laboratory research. Language Teaching Abstracts, 30, 73-87. doi: http://dx.doi.org/ $10.1017 / \mathrm{S} 0261444800012799$

Spada, N. (2011). Beyond form-focused instruction: Reflections on past, present and future research. Language Teaching, 44(2), 225-236. doi: http://dx.doi.org/10.1017/ S0261444810000224

Spada, N., \& Tomita, Y., (2010). Interactions between type of instruction and type of language feature: A meta-analysis. Language Learning, 60(2), 1-46. doi: 10.1111/j.14679922.2010.00562.x 
Swain, M. (1995). Three functions of output in second language learning. In G. Cook, \& B. Seidlhofer (Eds.), Principle and practice in applied linguistics (pp. 125-144). Oxford: Oxford University Press.

Uggen, M.S. (2012). Reinvestigating the noticing function of output. Language Learning, 62, 1-35. doi: 10.1111/j.1467-9922.2012.00693.x

VanPatten, B. (1996). Input processing and grammar instruction: Theory and research. Norwood, NJ: Ablex.

Williams, J., \& Evans, J. (1998). What kind of focus and on which forms? In C. Doughty \& J. Williams (Eds.), Focus on form in classroom second language acquisition (pp. 139-155). Cambridge: Cambridge University Press.

Yang, Y., \& Lyster, R. (2010). Effects of form-focused practice and feedback on Chinese EFL learners' acquisition of regular and irregular past tense forms. Studies in Second Language Acquisition, 32, 235-263. doi: http://dx.doi.org/10.1017/S0272263109990519 